\title{
Some Chaotic Points in Cuprate Superconductors
}

\author{
Özden Aslan Çataltepe \\ Anatürkler Educational Consultancy and Trading Company \\ Bă̆dat Cad. No: 258 3/6 Göztepe, İstanbul \\ Turkey
}

\section{Introduction}

The aim of this chapter is to determine the chaotic points of cuprate layered superconductors by means of magnetization data and the concept of the Josephson penetration depth based on Bean Critical State and Lawrance-Doniach Models, respectively. In this chapter, the high temperature mercury based cuprate superconductors have been examined by magnetic susceptibility (magnetization) versus temperature data, X-Ray Diffraction (XRD) patterns and Scanning Electron Microscope (SEM) outputs. Thus by using these data, a new method has been developed to calculate the Josephson penetration depth precisely, which has a key role in calculating various electrodynamics parameters of the superconducting system. The related magnetization versus temperature data have been obtained for the optimally oxygen doped virgin (uncut) and cut samples with the rectangular shape. By means of the magnetization versus temperature data of the superconducting sample, taken by Superconducting Quantum Interference Device (SQUID), the Meissner critical transition temperature, $T_{c}$, and the paramagnetic Meissner temperature $\mathrm{T}_{\mathrm{PME}}$, called as the critical quantum chaos points, have been extracted. In superconductors, the second order phase transition occurs at Meissner transition temperature, $T_{c}$, that is considered as the first chaotic point in the system, since the normal state of being is transformed into another state of being called as "superconducting state" that has been driven by temperature. The XRD measurements have been performed in order to calculate the lattice parameters of the system. The crystallographic lattice parameters of superconducting samples, determined by the XRD patterns, have been used to estimate the extent of the Josephson penetration depth. The SEM outputs have been used to determine the grain size of the optimally oxygen doped polycrystalline superconducting samples. The average grain size of the $\mathrm{HgBa}_{2} \mathrm{Ca}_{2} \mathrm{Cu}_{3} \mathrm{O}_{8+\mathrm{x}}(\mathrm{Hg}-1223)$ samples, $\mathrm{t}$, is a crucial parameter, since the critical current density value, $\mathrm{J}_{\mathrm{c}}$, is inversely proportional to " $\mathrm{t}$ ", whereas it is directly proportional to the difference in magnetization. It has been concluded that the grain size of the superconductors and the length of the c-axis of the unit cell of the system are highly effective on both of the first and second chaotic points of the superconducting system.

\section{The mercury based copper oxide layered superconductors}

It is well known that, the superconducting materials have a phase transition from normal state to superconducting state at the Meissner transition temperature, $\mathrm{T}_{\mathrm{c}}$. The most common 
property of the superconductivity is the diamagnetic response to the applied magnetic field. In addition to diamagnetic response, some superconductors exhibit a simultaneous paramagnetic behaviour under a weak applied magnetic field (Braunish et al., 1992; Braunish et al., 1993; Onbaşl1 et al., 1996; Nielsen et al., 2000). This paramagnetic behavior is called as Paramagnetic Meissner Effect (PME) and it can be observed within a specific temperature interval with the maximum paramagnetic signal at the paramagnetic Meissner temperature, $T_{\text {PME }}$. At this temperature, the direction of the orbital current changes its direction in the momentum space. Since both temperatures represent the transition from one state of being to another, $T_{c}$ and $\mathrm{T}_{\mathrm{PME}}$ are considered as the critical quantum chaos points of the superconducting specimens (Aslan et al., 2009; Onbaşl1 et al., 2009). The superconducting system is considered as the best material media displaying the chaotic behavior (Waintal et al., 1999; Bogomolny et al., 1999; Evangelou, 2001). The determination of the critical chaotic points is very important in order to decide about the operating temperatures for the high sensitive advanced technological applications. In this context, the determination of the critical chaotic points of $T_{c}$ and $\mathrm{T}_{\mathrm{PME}}$ on both a.c. (alternative current) and d.c. (direct current) magnetic susceptibility versus temperature data of the mercury based superconductors have been realized (Onbaşli et al., 1996; Aslan et al., 2009; Onbaşli et al., 2009).

The mercury based copper oxide layered superconductor investigated, which is one of the high temperature superconductors, has the highest critical parameters such as Meissner transition temperature, $\mathrm{T}_{\mathrm{c}}$, the critical current density, $\mathrm{J}_{\mathrm{c}}$, etc. (Onbaşli et al., 1996; Aslan et al., 2009). Due to the highest critical parameters of the bulk superconducting Hg-1223 samples, the determination of some electrodynamics parameters such as Josephson penetration depth, plasma frequency and the anisotropy factor has also a great importance for both theoretical and various advanced technological applications. To calculate these electrodynamics parameters, the average spacing of copper oxide bilayers, s, and the grain size of the superconductor are required to be measured. The average spacing of copper oxide bilayers, $\mathrm{s}$, is seen in the primitive cell of the mercury cuprate superconductors given in Fig. 1.

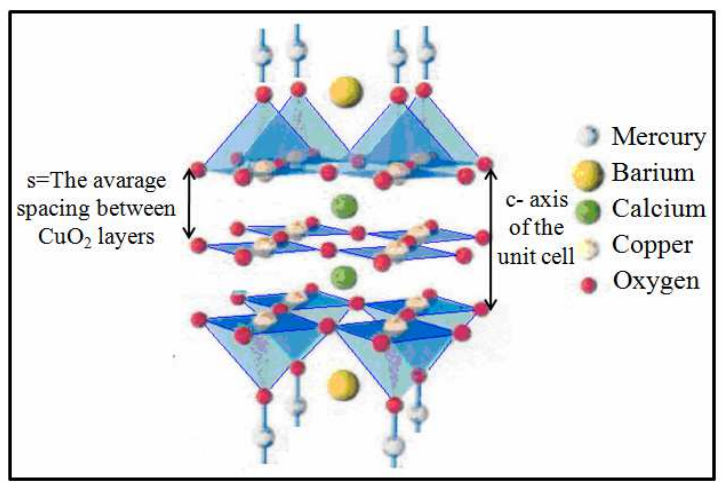

Fig. 1. The primitive cell of $\mathrm{Hg}-1223$ superconductor at the normal atmospheric pressure (Aslan, 2007).

The primitive cell of the mercury cuprates contains three superconducting copper oxide planes separated by insulating layers and this structure is considered as an intrinsic Josephson junction array (Fig. 1). 
As is known, the superconductivity occurs in the copper oxide planes which form intrinsic structural layers. The origin of the superconductivity is based on the harmony which is extended to all copper oxide layers along the c-axis via electromagnetic coupling at the Josephson plasma frequency, $\omega_{\mathrm{p}}$ (Lawrance \& Doniach, 1971). However, the Josephson penetration depth, $\lambda_{j}$, being the most important electrodynamics parameters, is given in Eq. (1)

$$
\lambda_{j}=\sqrt{\frac{\Phi_{o} \mathcal{c}}{2 \pi J_{c} \mu_{O} s}}
$$

where $\Phi_{0}=2.0678 \times 10^{-15}\left(T^{2} m^{2}\right)$ represents the flux quantum, $c$ is the velocity of light, $\mu_{o}=4 \pi \times 10^{-7}\left(\frac{N}{A^{2}}\right)$ is the permeability of free space, $\mathrm{J}_{\mathrm{c}}$ is the critical current density and $\mathrm{s}$ is the average spacing of copper oxide bilayers. According to the scientific literature, the Josephson penetration depth, $\lambda_{j}$, is considered as a measure of the magnetic penetration depth of the field induced by super current (Gough, 1998; Ketterson \& Song, 1999; Tinkham, 2004; Fossheim \& Sudbo, 2004). It has been previously determined that the Josephson penetration depth, $\lambda_{j}$ increases with temperature for the mercury cuprate superconducting family (Özdemir et al., 2006; Güven Özdemir et al, 2007).

In the next section, both the required lengths and quantum chaotic points mentioned above for the bulk superconducting Hg-1223 samples will be examined by means of XRD patterns, SEM outputs and magnetic moment versus temperature data.

\section{Determination of the chaotic points}

\subsection{The analysis of temperature dependence of magnetization}

The concept of chaos can be defined as the transition from one state of being to another state of being where the probability density of the system, which is sensitive to the initial conditions, changes via temperature (Gleick, 1987; Panagopoulos \& Xiang, 1998). In this point of view, the superconducting system is one of the best examples to understand the unexpected chaotic transitions via magnetic measurements. Superconducting systems, which exhibit the second order phase transition, possess some critical chaotic points as defined above. According to many researchers, the phenomenon of the critical quantum chaos have been observed in the quasi periodic systems, the systems with two interacting electrons and the fractal matrices (Evangelou \& Pichard, 2000; Evangelou, 2001). Furthermore, the superconductors investigated, in which phonon mediated attractive electron-electron interaction leads to form quasi-particles, namely Cooper pairs (Aoki et al, 1996; Egami et al., 2002; Tsudo \& Shimada, 2003), constitute a natural laboratory for searching and observing quantum critical chaotic points (Onbaşlı et al., 2009).

In this section, the optimally oxygen doped superconducting samples have been investigated by referring to $T_{c}$ and $T_{P M E}$ temperatures extracted from the magnetic susceptibility versus temperature data taken by Quantum Design SQUID susceptometer model MPMS-5S. In all of the magnetization measurements, the magnetic field has been applied to the superconducting bulk specimen along the c-axis.

The optimally doped virgin (uncut) samples have been obtained by pressing under 1 ton of weight. Hg-1223 samples, which have been kept in air for several months after being synthesized, were still mechanically very hard, dense and stiff (Onbaşl1 et al., 1996; Onbaşl1 et al., 1998; Güven Özdemir et al., 2009). Afterwards, the virgin samples have been cut by 
diamond saw in the rectangular shape of $4 \times 2 \times 1 \mathrm{~mm}$. Hence the magnetic susceptibility of the $\mathrm{Hg}$ based cuprate superconducting samples has been investigated under both a.c. and d.c. magnetic fields (Onbaşlı et al., 1996; Onbaşl1, 2000).

The related a.c. data for the optimally doped virgin (uncut) and cut samples, which belong to the same virgin batch, are given in Fig. 2. Both data have been taken under a.c. magnetic field of 1 Gauss with $1 \mathrm{kHz}$ frequency.

As seen in Fig. 2, the paramagnetic Meissner and the critical Meissner chaotic temperatures of the uncut samples have been determined as $126 \mathrm{~K}$ and $137 \mathrm{~K}$, respectively. However, for the cut samples with rectangular shape, $T_{\mathrm{PME}}$ and $\mathrm{T}_{\mathrm{c}}$ have been found as $122 \mathrm{~K}$ and $140 \mathrm{~K}$, respectively. As is known that the paramagnetic Meissner effect can also be observed on very cleanly prepared polycrystalline samples under d.c. magnetic fields. Magnetic moment versus temperature curve for the uncut sample has been taken under zero and 1 Gauss of d.c. magnetic field. The paramagnetic Meissner effect has been observed under d.c. field cooled data of the uncut (virgin) specimen (Fig. 3).

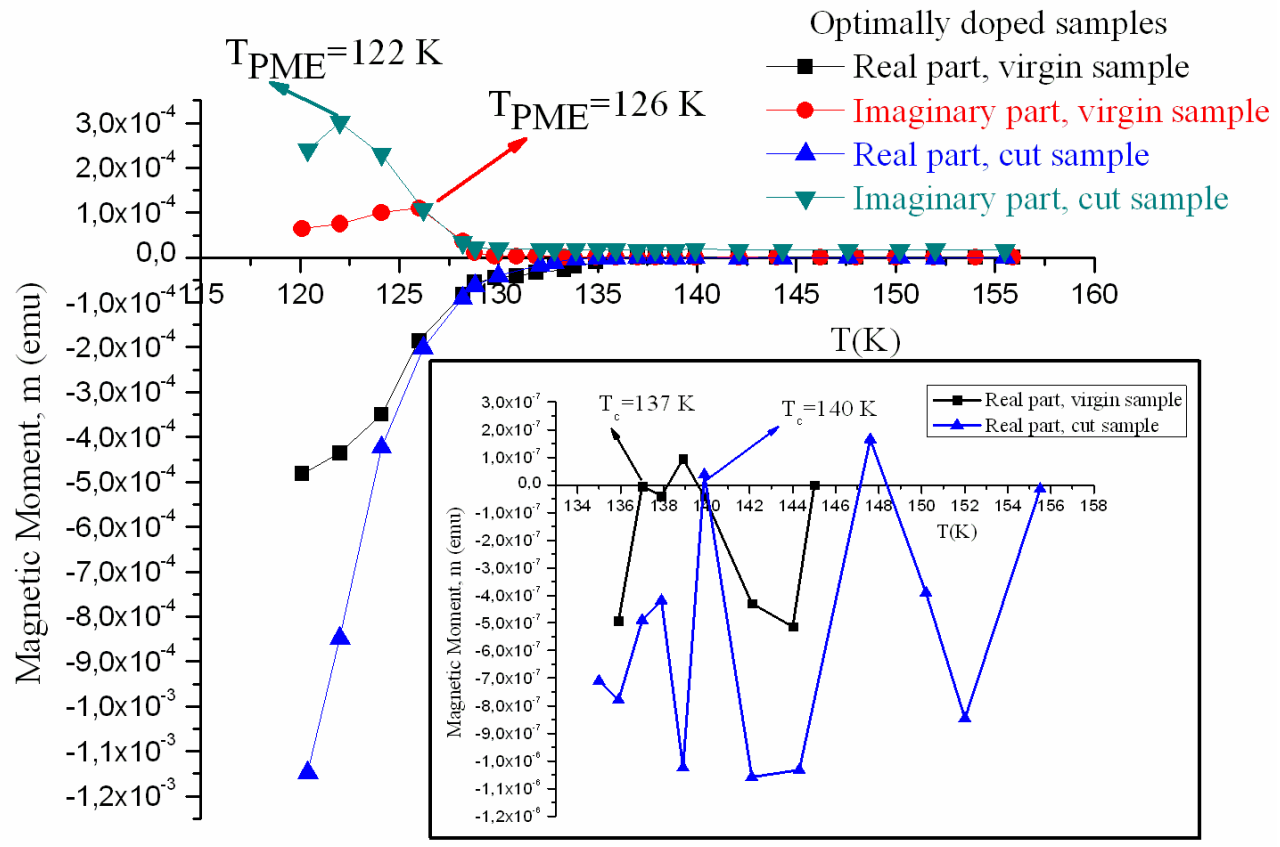

Fig. 2. Magnetic moment versus temperature curves of the virgin (uncut) $\mathrm{Hg}-1223$ and cut samples under a.c. magnetic field of 1 Gauss. The inset shows the real part of the magnetic moment and indicates the Meissner critical temperatures for both of the virgin and cut specimens.

\subsection{The symmetries and symmetry breakings in $\mathrm{Hg}-1223$ superconductors}

The concepts of the symmetries and symmetry breakings are accepted as one of the most unsolved problems of the 21st century. The symmetries have a crucial role in giving information about the present forces in a system considered and that symmetries can be broken in various ways such as variation of density, temperature, etc. (Nambu \& Pascual, 1963; Smolin, 2006). 
The concept of symmetry breakings has been discussed by the phenomenon of the critical quantum chaos in the mercury cuprates by means of the magnetic susceptibility versus temperature graphics.

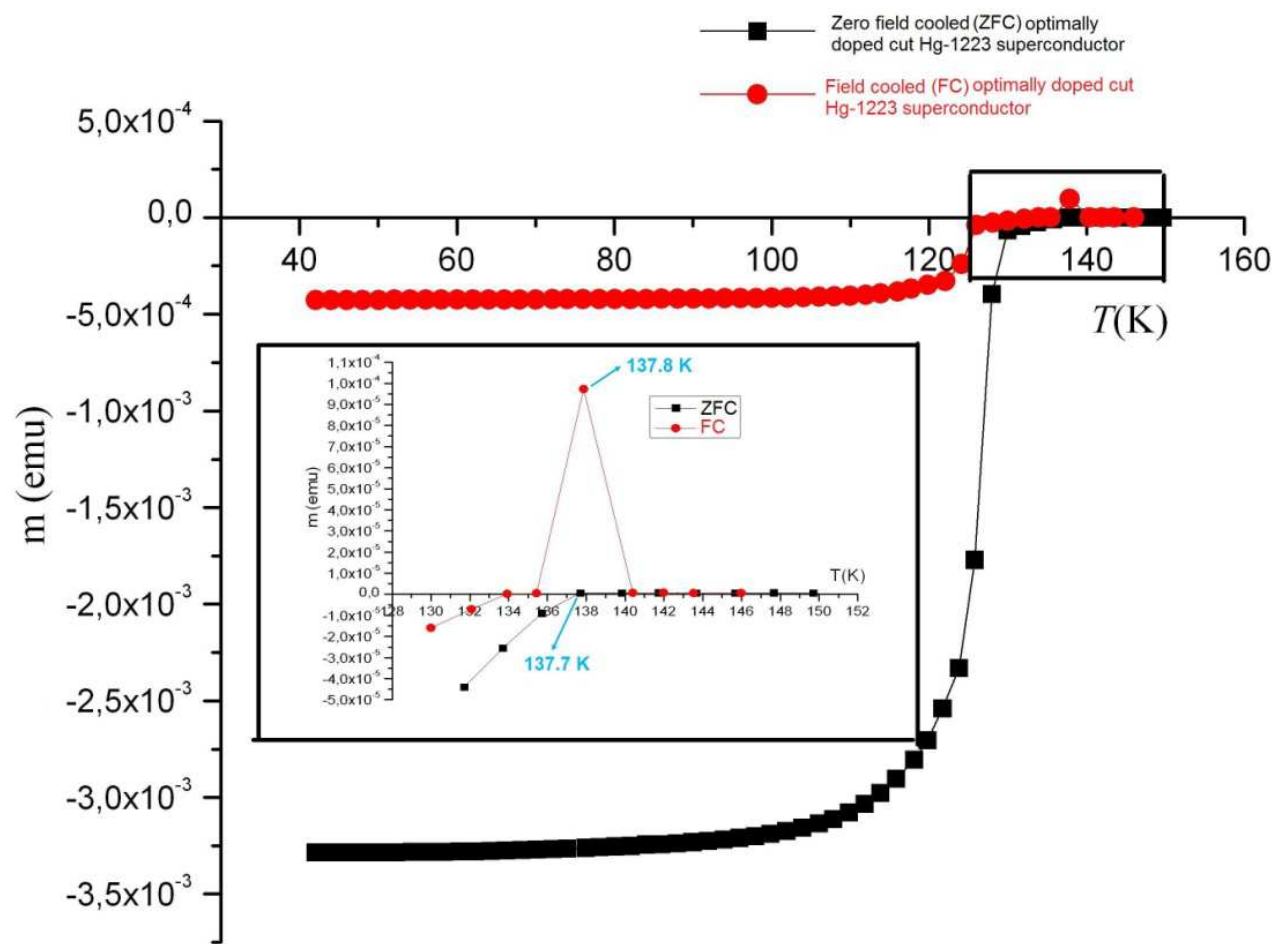

Fig. 3. Magnetic moment versus temperature curves of the optimally doped uncut (virgin) Hg-1223 superconductors under zero and 1 Gauss d.c. magnetic field cooled. The inset shows that only the field cooled specimen displays PME.

The global gauge symmetry is broken at Meissner transition temperature, $\mathrm{T}_{\mathrm{c}}$, in high temperature superconductors (Zhang, 2001; Li, 2003; Roman, 2004; Onbaşlı et al., 2009). Accompanying the global gauge symmetry breaking, the symmetry of the order parameter undergoes a transition from s-wave to d-wave at $T_{c}$, as well. Furthermore, due to the fact that the system exhibits spatial Bose-Einstein condensation (Güven Özdemir et al., 2007), the superconducting system can be considered to display f-wave symmetry, as well. The schematic representations of the s-wave, $\mathrm{d}$-wave and $\mathrm{f}$-wave symmetries are given in Fig. 4 . Moreover, Weinberg states that "A superconductor is simply a material in which electromagnetic gauge invariance is spontaneously broken." With this statement, Weinberg means that the electromagnetic gauge field acquires a mass due to the Higgs mechanism in a superconductor. In other words, the particle physicists often speak of gauge invariance interchangeably with the Higgs mechanism (Weinberg, 1996; Greiter, 2005).

In addition to this symmetry breaking at $T_{c}$, the time reversal symmetry breaking phenomenon becomes observable on paramagnetic Meissner effect at $\mathrm{T}_{\mathrm{PME}}$ in mercury cuprates. In the unconventional (high temperature) superconductors, the breaking of the 
time reversal symmetry is related to the orbital magnetism. The origin of the PME has been estimated by the reversion mechanism of the direction of the orbital current (Li, 2003; Onbaşli et al., 2009). According to Sigrist et al., the time reversal symmetry can be destroyed by application of magnetic field and that addition of magnetic impurities (Sigrist et al., 2006). In scientific literature, it has been predicted that the time reversal symmetry breaking occurs below Meissner transition temperature, $\mathrm{T}_{\mathrm{c}}$ in superconductors (Horovitz \& Golub, 2002).
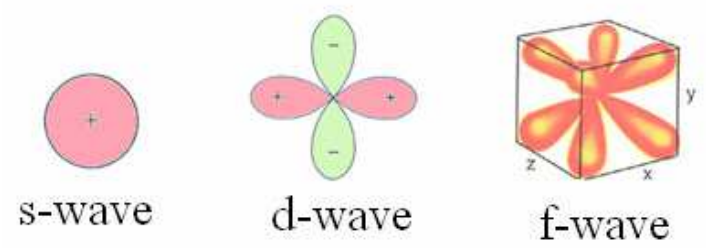

Fig. 4. The schematic representation of s-wave, $d$-wave and f-wave.

The PME phenomenon has been suggested as a reliable method for determining broken time reversal symmetric state in superconductors instead of very complicated experimental methods such as the angle resolved photoelectron spectroscopy (ARPES) (Onbaşli et al., 2009). Using ARPES for detecting time reversal symmetry breaking phenomenon may bring the possibility of having the order parameter to be collapsed. So that the magnetic method introduced in this chapter will be a reliable tool to detect the symmetry breaking points of the high $\mathrm{T}_{\mathrm{c}}$ superconductors (Onbaşli et al, 2009).

In recent years, it has been suggested that the copper oxide layered superconductors are considered as a perfect prototype for the electroweak theory and electroweak symmetry breaking due to Higgs mechanism in superconductors (Quigg, 2008). The Higgs mechanism in layered superconductors has been explained by Josephson plasma excitations. In weakly Josephson coupled layered superconductors, the main Josephson plasma excitation modes consist of the longitudinal and transversal modes. The transversal Josephson plasma excitation is an electromagnetic wave, propagating perpendicular to the polarization vector (a-Tachiki et al., 1996; b-Tachiki et al., 1996). On the other hand, the longitudinal mode known as Nambu-Goldstone (Anderson-Bogalibov) mode is an elementary excitation mode accompanying with the superconducting phase transition due to the symmetry breaking (Anderson, 1958; Rickazyen, 1958; Nambu, 1960). However, the zero energy gap at $\mathrm{k}=0$, does not obey to the Goldstone theorem. Therefore, an additional mechanism, which is known as Higgs mechanism, has been suggested to obtain the finite energy gap. In this point of view, the longitudinal plasma waves should be massive since Higgs bosons have finite mass (Kadowaki et al., 1998). As is known that, all the electroweak force particles are massless in the electroweak symmetry. On the other hand, the breaking of the electroweak symmetry gives mass to the electroweak force particles $\mathrm{W}^{ \pm}$and $\mathrm{Z}^{0}$ (namely weak gauge bosons) leaving the photon massless (Quigg, 2006). According to Veltman, if the space is filled with a type of superconductor, it gives mass to $\mathrm{W}^{ \pm}$and $\mathrm{Z}^{0}$ bosons (Goldstone bosons). This superconductor can be considered as consisting of Higgs bosons (Veltman, 1986). It has been proposed that Higgs boson has zero spin and zero angular momentum. It has been predicted that, the time reversal symmetry breaking at PME temperature, in which the angular momentum is zero, can be considered as the emerging of Higgs boson in the superconducting state (Onbaşl1 et al., 2009). 
Anderson discovered the physical principle underlying the formation of mass mechanism in the context of superconductivity. The boson, which appears as a result of the Goldstone theorem, has zero unrenormalized mass, which is converted into a finite mass plasmon by interaction with electromagnetic gauge field (Anderson, 1963). The effective mass of the quasi particles, $\left(\mathrm{m}^{*}\right)$, introduced in the following chapter, corresponds to the three dimensional (spatial) net effective mass, which is neither attributed to Goldstone boson nor plasmon. In the following chapter, the third quantum chaotic point will be introduced. The third quantum chaotic point called as, quantum gravity point, $\mathrm{T}_{\mathrm{QG}}$, where the net effective mass of the quasi-particles $\left(\mathrm{m}^{*}\right)$ in the superconducting system has the maximum value, corresponds to the quantum gravity peak for the optimally oxygen doped mercury cuprate superconductors, at which the plasma frequency shifts from microwave to infrared region at the $\mathrm{T}_{\mathrm{QG}}$ temperature (Aslan Çataltepe et al., 2010).

\subsection{Relevant distribution functions of the mercury based superconductors}

At $T_{c}$, the distribution functions of the system differs from one to another while the transition from normal state to superconducting state occurs. Hence, the system includes both Fermi Dirac (F-D) and Bose-Einstein (B-E) distribution functions depending on the normal state and superconducting state, respectively. As the temperature is higher than the Meissner transition temperature, $\mathrm{T}_{\mathrm{c}}$, the system is in its normal state that obeys to F-D distribution. So that the partition function yields to $\left(\mathrm{e}^{A+1}\right)$. The shorter presentation of the exponential term contained by both of the distribution functions is abbreviated by $A=(E$ $\mu) / \kappa_{B} T$, where $E$ is the energy of the system, $\mu$ is the chemical potential, $\kappa_{B}$ is the Boltzmann constant and $T$ is the temperature. At normal state, where $T>T_{C}$, the spin quantum number (S) is $1 / 2$ and angular momentum quantum number $(\mathrm{L})$ is zero.

At the critical transition temperature, $T=T_{c}$, the exponential term becomes equal to 1 (unity)

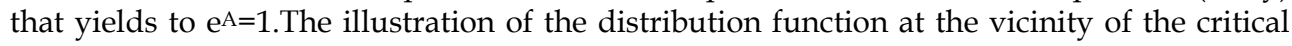
Meissner transition temperature is given in Fig. 5.

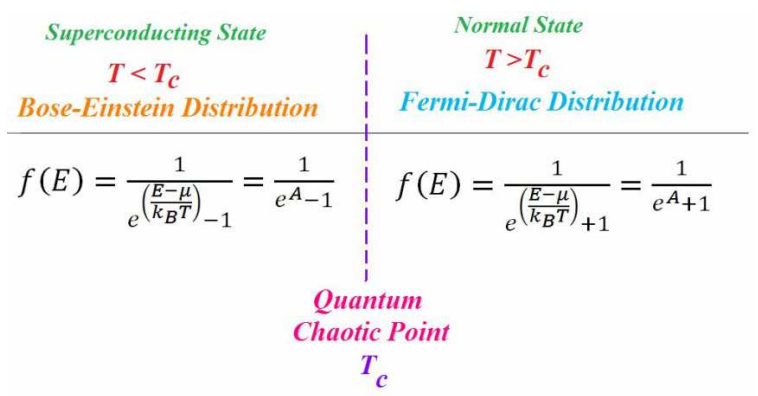

The partition function equals to zero.

$$
\begin{gathered}
e^{A}-1=0 \\
e^{\left(\frac{E-\mu}{k_{B} T}\right)}=1 \\
E-\mu=0 \\
E=\mu
\end{gathered}
$$

Fig. 5. The repsentative illustration of the distribution function at the vicinity of the critical Meissner temperature $\mathrm{T}_{\mathrm{c}}$. At the Meissner transition temperature, the partition function equals to zero that results in the equality of the chemical potential to the total energy of the system. 
At $\mathrm{T}_{\mathrm{c}}$, the absolute value of the chemical potential, $\mu$ equals to the total energy of the system, $E$. Hence, the partition function approaches to zero, so that the distribution function diverges to infinity.

Below $\mathrm{T}_{\mathrm{c}}$, the distribution function obeys to B-E distribution where the angular momentum and spin quantum numbers are $L=2$ and $S=0$, respectively.

In short, the exponential terms of the partition functions both have the same magnitude that reaches the unity at $\mathrm{T}=\mathrm{T}_{\mathrm{c}}$. So that the \pm 1 interval has a crucial role for determining the distribution functions and that of the order parameters of the superconducting system, as well (Onbaşlı et al., 2006).

\subsection{The quantum mechanical analysis of mercury cuprate superconductors}

The quantum mechanical interpretation of PME is based on the development of a conceptual relationship between the time reversal symmetry and the magnetic quantum number of the system. It is known that, reversing the time $(t)$ not only replaces $t$ by $-t$ in equations, but also it reverses momentums defined by the time derivatives of spatial quantities such as angular momentum, L. Furthermore, magnetic quantum number, m, refers to the projection of the angular momentum, $\mathrm{L}_{\mathrm{z}}$. This component of angular momentum in $\mathrm{z}$ direction is defined by the well known formula:

$$
\mathrm{L}_{\mathrm{z}}=\mathrm{m} \hbar
$$

where $\hbar(=\mathrm{h} / 2 \pi)$ is the reduced Planck constant. Since, there is a relationship between the magnetic moment and the magnetic quantum number, inverting the direction of the time flow will affect the sign of the $\mathrm{z}$ component of the angular momentum, the magnetic quantum number, and magnetic moment of the system. For this reason, the magnetic moment (susceptibility ${ }^{1}$ ) versus temperature data has been re-examined in the context of magnetic quantum numbers as illustrated in Fig. 6. In this respect, alternative current magnetic susceptibility versus temperature data of the optimally oxygen doped $\mathrm{Hg}$-based cuprate had been previously suggested to explain the time reversal symmetry breaking phenomenon (Onbaşlı et al., 2009). In Fig. 6, magnetic susceptibility versus temperature curve of Hg-1223 has been divided into three regions with respect to magnetic quantum number, $\mathrm{m}$. Since the system investigated is represented by the d-wave symmetry with the orbital quantum number, $\ell$, equals to 2 , the $m$ values will vary from minus two to plus two $(\ell=2, \mathrm{~m}= \pm 2, \pm 1,0)$.

In the non-superconducting region III, the superconducting system has the room temperature symmetry (s-wave symmetry). The temperature region, at which the d-wave symmetry is valid, has been divided into two parts. In region II, magnetic quantum number, $\mathrm{m}$, equals to \pm 1 . Since the imaginary component of the magnetic susceptibility is related to the losses of the system, the imaginary component of magnetic susceptibility in region II corresponds to the $m=-1$ domain. Hence the real component of magnetic susceptibility in region II corresponds to $\mathrm{m}=+1$ domain. Furthermore, $\mathrm{m}$ is equal to minus and plus two in region I. By reducing the temperature, the magnetic quantum number of the system experiences a change from "-" to "+" and vice versa. This means that the projection of the

${ }^{1}$ Magnetic measurements have been performed under 1 Gauss of magnetic field. 
angular momentum in $\mathrm{z}$ direction, $\mathrm{L}_{\mathrm{z}}$, passes through "zero" at $\mathrm{T}_{\mathrm{PME}}$. From this point of view, $\mathrm{T}_{\mathrm{PME}}$ is attributed to the breaking point of the time reversal symmetry.

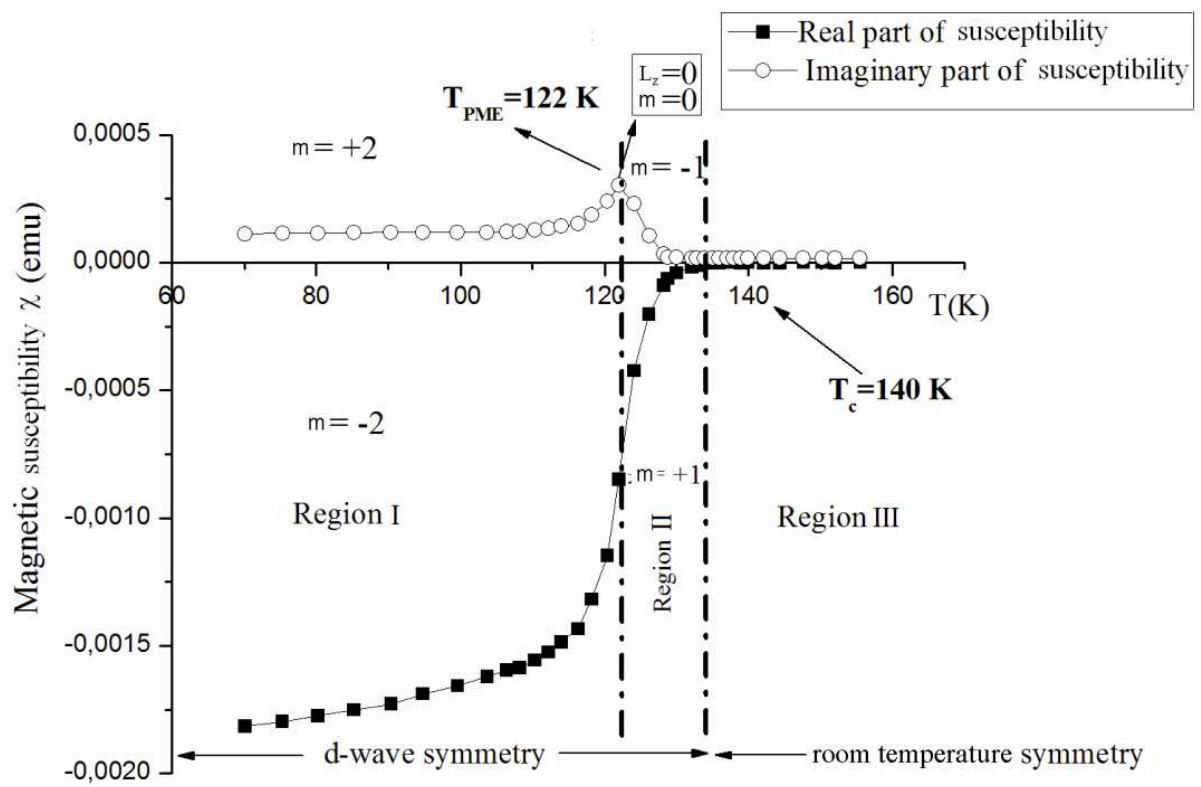

Fig. 6. Magnetic susceptibility (magnetic moment) versus temperature for the optimally oxygen doped Hg-1223 sample at 1 Gauss of a.c. magnetic field. The d-wave and room temperature symmetry regions together with the related magnetic quantum numbers $(\mathrm{m})$ are indicated for three distinguished regions (Onbaşlı et al., 2009).

Related to the quantum mechanical analysis, the concept of the parity should be taken into account. For $\mathrm{T}>\mathrm{T}_{\mathrm{PME}}$ temperatures, the superconducting system has the odd parity. In the other words, the wave function of the system is anti-symmetric, so there is 2-dimensional degree of freedom. For the temperatures lower than $\mathrm{T}_{\mathrm{PME}}$, the superconducting system has the even parity and the symmetric wave function with 1-dimensional degree of freedom.

Moreover, it has been determined that, the quantum gravity point, $\mathrm{T}_{\mathrm{QG}}$, which emphasized in the following chapter, appears at region I at which the superconducting system has one dimensional degree of freedom with the even parity.

\section{X-Ray Diffraction (XRD) pattern analysis and the lattice parameters of the mercury cuprates}

In this work, the crystal structure of the mercury based copper oxide layered high temperature superconductors is determined by the XRD measurements. The first motivation of performing the XRD measurements is to determine the " $\mathrm{s}$ " parameter, which has a crucial importance in calculating the Josephson penetration depth electrodynamics parameter. The second motivation is to investigate the effects of the crystal structure of the superconducting sample on the critical quantum chaos points. As is known, the crystal structure is directly affect the critical quantum chaos point temperatures, such as $\mathrm{T}_{\mathrm{c}}$ and $\mathrm{T}_{\mathrm{PME}}$ (Aslan et al., 2009). 
The XRD patterns have been extracted from a Cu/40kV /40kA Rigaku Model XRD device. The XRD patterns of the optimally doped and under oxygen doped samples have been shown in Fig. 7 and Fig. 8, respectively.

According to Fig. 7 and Fig. 8, the lattice parameters of both the optimally and under doped Hg-1223 superconductors have been calculated and the results are given in Table 1 and Table 2, respectively (Aslan, 2007; Aslan et al., 2009).

\begin{tabular}{|c|c|c|}
\hline a-axis & b-axis & c-axis \\
\hline $3.8684 \dot{A}$ & $3.8684 \dot{A}$ & $15.7182 \dot{A}$ \\
\hline
\end{tabular}

Table 1 . The lattice parameters of the optimally doped sample calculated from the XRD data given in Fig. $7\left(\mathrm{~T}_{\mathrm{c}}=140 \mathrm{~K}\right)$.

\begin{tabular}{|c|c|c|}
\hline a-axis & b-axis & c-axis \\
\hline $3.8328 \dot{A}$ & $3.8328 \dot{A}$ & $15.7452 \dot{A}$ \\
\hline
\end{tabular}

Table 2. The lattice parameters of the under doped sample calculated from the XRD data given in Fig. $8\left(\mathrm{~T}_{\mathrm{c}}=135 \mathrm{~K}\right)$.

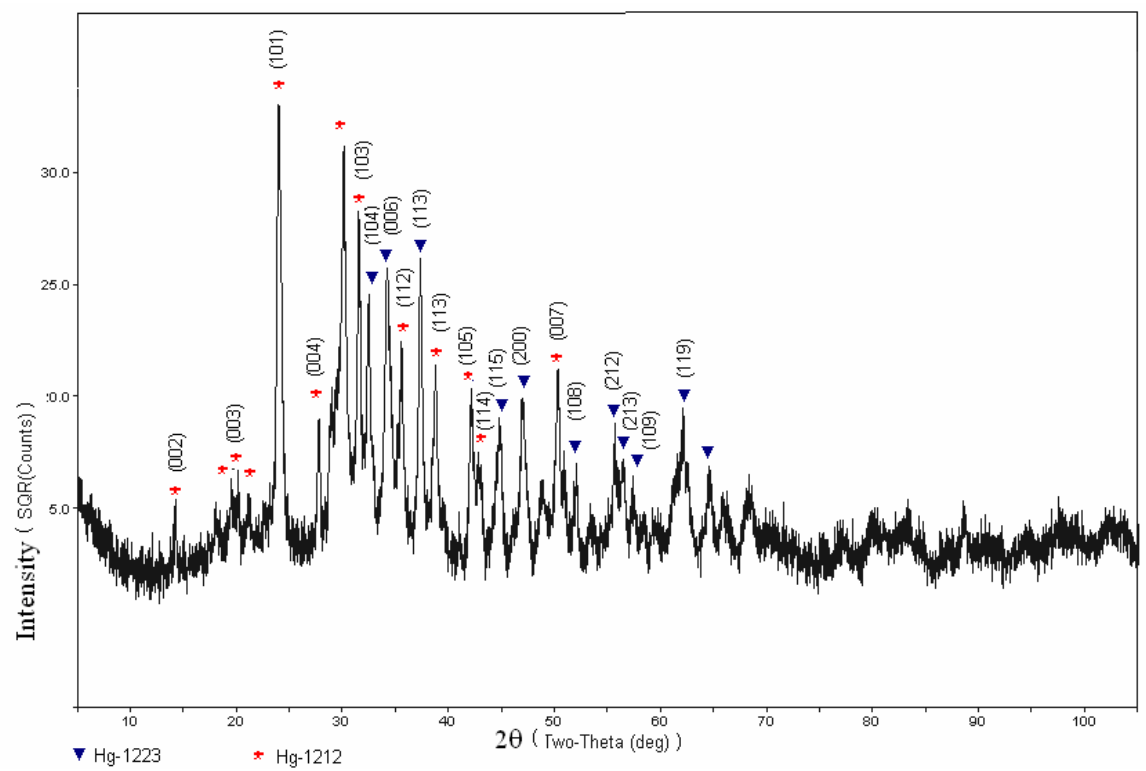

Fig. 7. XRD Pattern and (hkl) planes (Miller indices) of the optimally doped sample in 50 minutes counting. * indicates $\mathrm{Hg}-1212$ and $\boldsymbol{\nabla}$ indicates $\mathrm{Hg}-1223$ phases, respectively.

The X-ray data taken on the optimally and under-doped samples have been clearly shown a mixed phase of $\mathrm{HgBa}_{2} \mathrm{CaCu}_{2} \mathrm{O}_{6+x}(\mathrm{Hg}-1212)$ and $\mathrm{HgBa}_{2} \mathrm{Ca}_{2} \mathrm{Cu}_{3} \mathrm{O}_{8+x}(\mathrm{Hg}-1223)$. According to the data taken on both samples, the crystal symmetries have been found to be tetragonal structure with a space group of $\mathrm{P} 4 / \mathrm{mmm}$. This result is also consistent with the previous scientific literature on the crytal symmetry of mercury cuprate family superconductors. As is known, 
that the crystal structure of cuprates can generally be divided into two categories; tetragonal and orthorhombic lattices. Some cuprates such as $\mathrm{La}_{2-\mathrm{x}} \mathrm{Sr}_{\mathrm{x}} \mathrm{CuO}_{4}, \mathrm{Tl}_{2} \mathrm{Ba}_{2} \mathrm{CaCu}_{2} \mathrm{O}_{8}$, $\mathrm{HgBa}_{2} \mathrm{CaCu}_{2} \mathrm{O}_{6}(\mathrm{Hg}-1212)$, and $\mathrm{HgBa}_{2} \mathrm{Ca}_{2} \mathrm{Cu}_{3} \mathrm{O}_{8+x}$ (Hg-1223) and some others have the tetragonal crystal structure (Wagner et al., 1995; Gough, 1998; Tsuei \& Kirtley, 2000; Li, 2003).

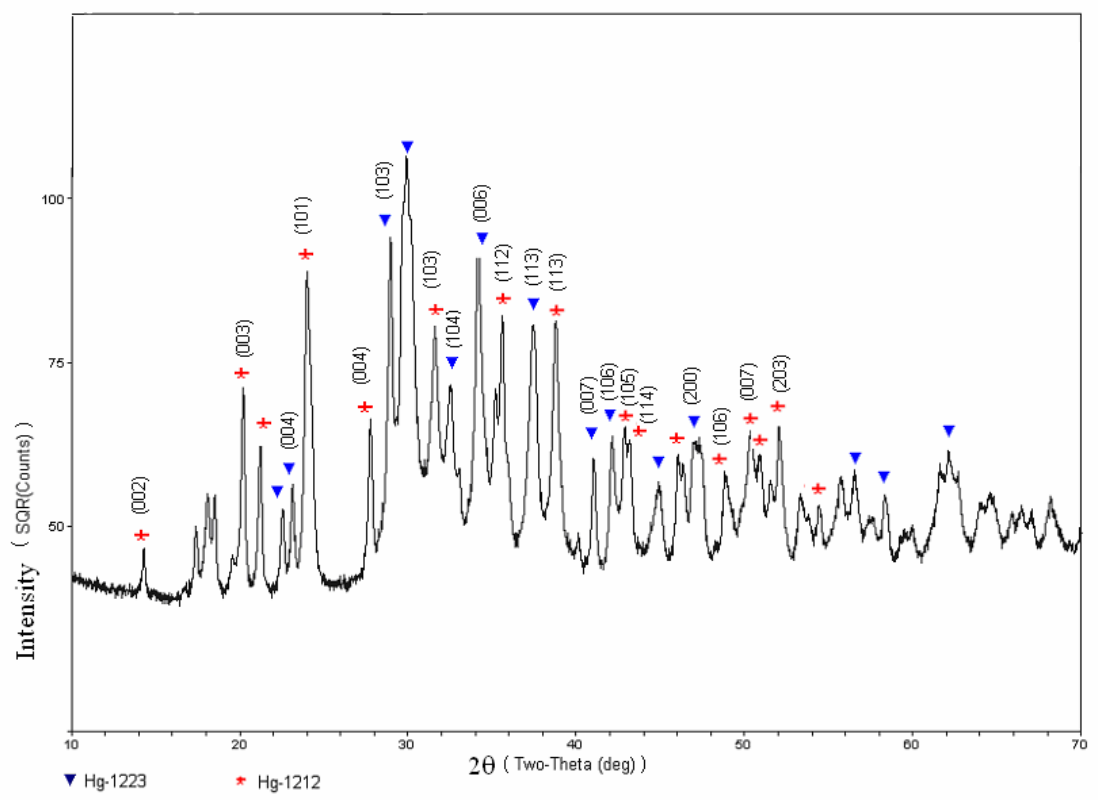

Fig. 8. XRD Pattern and (hkl) planes (Miller indices) of the under doped sample in 7 hours counting. * indicates $\mathrm{Hg}-1212$ and $\boldsymbol{\nabla}$ indicates $\mathrm{Hg}-1223$ phases, respectively.

The average spacing between the copper oxide layers of the mercury based sample, " $\mathrm{s}$ " has been determined from the translation vector along the c-direction of the unit cell2.

The average spacing between copper oxide layers of the optimally and the under oxygen doped samples have been calculated as $7.8591 \dot{A}$ and $7.8726 \dot{A}$, respectively. Both of the average spacing values will be used to determine the Josephson penetration depth of the superconductors in further works.

Moreover, it has been determined that the superconducting plane (ab-plane) of the optimally doped sample is larger than that of the under-doped sample. However, the lattice parameter along the c-axis of the optimally doped sample is $0.027 \dot{A}$ shorter than the other one. Recalling the fact that the reduction in c-axis parameter increases the quantum tunnelling probability between the superconducting $\mathrm{CuO}_{2}$ ab-planes, so that the $\mathrm{T}_{\mathrm{c}}$ of the optimally doped sample is higher than that of the under-doped sample. According to our experimental studies, it has been determined that the deficiency of oxygen doping reduces the $T_{c}$ by few Kelvin degree for bulk mercury based sample (Onbaşli et al., 1996). Also, it has been observed that the critical quantum chaos points of the optimally doped sample are higher than that of non-doped samples.

2The schematic representation of the unit cell of $\mathrm{Hg}-1223$ superconductors was given in Fig. 1. 
Furthermore, as is known, the existence of the intrinsic Josephson effect in superconductors indicates the formation of natural super lattices of Josephson junctions in the crystal structure. According to Ustinov, the spatial period of the super lattices is only $15 \dot{A}$ so that Josephson junctions are densely packed in the intrinsic structure (Ustinov, 1998). From this point of view, the mercury based copper oxide layered superconductors also include a natural super lattice with the translation vector magnitude of $15.71 \dot{A}$ and $15.74 \dot{A}$ for the optimally and under doped samples, respectively.

\section{The Scanning Electron Microscope (SEM) analysis and the average grain size of the superconducting samples}

In this work, the average grain size " $\mathrm{t}$ " of the mercury based copper oxide layered high temperature superconductors is determined by the SEM measurements. The motivation of performing the SEM measurements is to determine the " $\mathrm{t}$ " parameter, which has a crucial importance in calculating the critical current densities, $\mathrm{J}_{\mathrm{c}}$ and the interrelated parameter of Josephson penetration depth, $\lambda_{\text {j }}$.

The critical current density of the mercury cuprates is calculated by the Bean Critical State Model (Bean, 1962; Bean, 1964) below the lower critical magnetic field 3 ,

$$
J_{c}=30 \frac{4 \pi \Delta M}{t}
$$

where $\Delta M$ is the magnetization difference between the increasing and decreasing field branches of the $\mathrm{M}-\mathrm{H}$ curves and $\mathrm{t}$ is the average grain size of the specimen (Onbaşli et al., 1998). The dynamic hysteresis measurements of the optimally oxygen doped Hg-1223 samples for various temperatures have been performed by the Quantum Design SQUID susceptometer, model MPMS-5S (Fig. 9). The magnetic field of 1 Gauss has been applied parallel to the c-axis and the critical currents flowed in the ab-plane of sample.

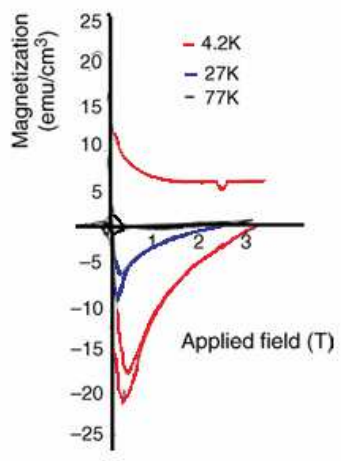

Fig. 9. Magnetization versus applied magnetic field (M-H) for the optimally oxygen doped Hg-1223 samples at 4.2, 27 and $77 \mathrm{~K}$.

${ }^{3}$ Since the calculations have been made below the lower critical magnetic field, $\mathrm{H}_{\mathrm{c} 1}$, the whole magnetic flux has been totally expelled from the sample. 
The SEM measurements have been performed by JSM-5910 LV and ESD X350 model SEM devices. The related SEM images are given in Fig. 10 and Fig. 11.

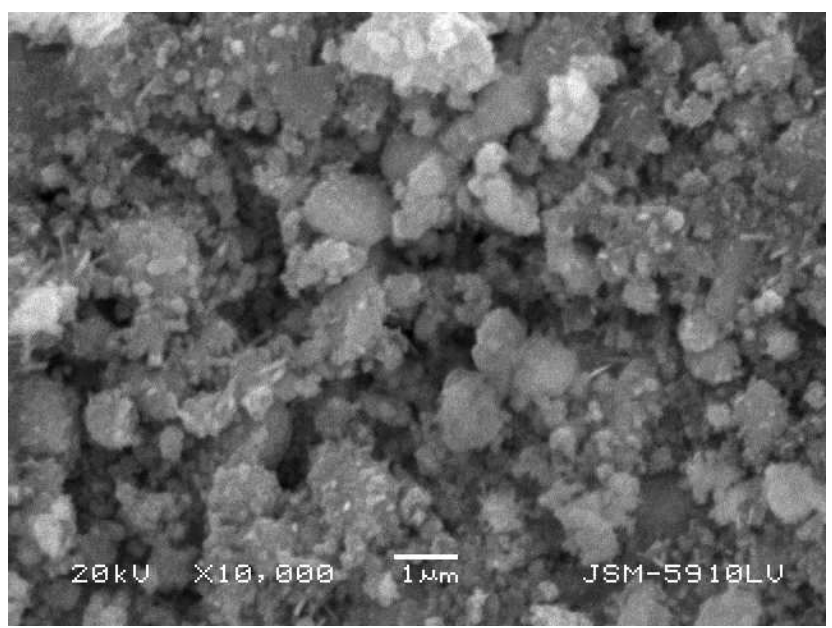

Fig. 10. The optimally oxygen doped $\mathrm{Hg}-1223$ sample with rectangular shape. The SEM output has been taken at $20 \mathrm{kV}$ and JSM-5910 LV $\left(\mathrm{T}_{\mathrm{c}}=140 \mathrm{~K}, \mathrm{t}=1.098 \mu \mathrm{m}\right)$

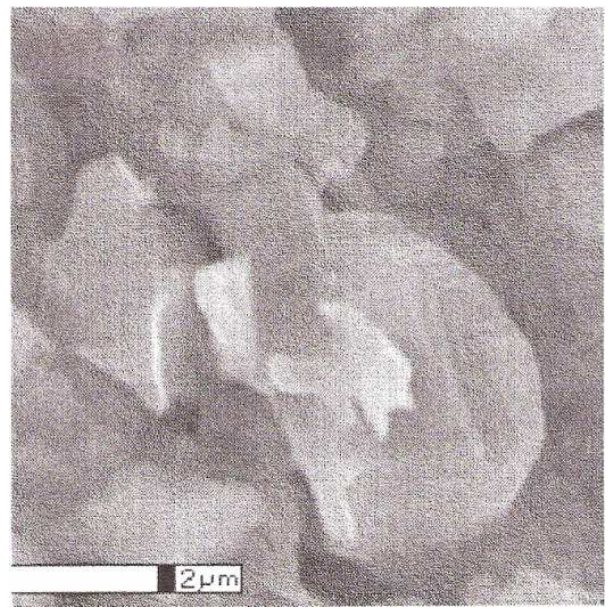

Fig. 11. The optimally oxygen doped uncut (virgin) Hg-1223 sample. The SEM output has been taken at $20 \mathrm{kV}$, ESD X350. $\left(\mathrm{T}_{\mathrm{c}}=136 \mathrm{~K}, \mathrm{t}=1.5 \mu \mathrm{m}\right)$ (Onbaşli et al, 1998).

The average grain sizes of the Hg-1223 samples have been found by using the intercept method and the results are given in Table 3.

According to the SEM outputs, the grain size of the superconductors affects the Meissner transition temperature which is the one of the critical quantum chaos points. In experimental studies, it has been found that the smaller the grain size the higher the Meissner transition temperature (Table 3) (Aslan et al., 2009; Özdemir et al., 2006; Onbaşlı et 
al., 1998). Also, the effect of the shape of the superconductors has been investigated by the magnetic moment versus temperature data by which the critical quantum chaos points have been determined (Table 3) (Aslan et al., 2009).

\begin{tabular}{|c|c|c|c|}
\hline & $\begin{array}{c}\text { The grain size, } \\
\mathrm{t}(\mu \mathrm{m})\end{array}$ & $\begin{array}{c}\text { The Meissner transition } \\
\text { temperature, } \\
\mathrm{T}_{\mathrm{c}}(\mathrm{K})\end{array}$ & $\begin{array}{c}\text { The paramagnetic } \\
\text { Meissner temperature, } \\
\mathrm{T}_{\mathrm{PME}}(\mathrm{K})\end{array}$ \\
\hline Cut sample & 1.098 & 140 & 122 \\
\hline Virgin sample & 1.5 & 137 & 126 \\
\hline
\end{tabular}

Table 3. The grain size and the Meissner transition temperatures of cut and virgin samples

By using Eq. (3) and the grain sizes determined by SEM, the critical current densities for the optimally doped $\mathrm{Hg}-1223$ superconductors have been calculated to vary $10^{12} \mathrm{~A} / \mathrm{m}^{2}$ to $10^{10}$ $\mathrm{A} / \mathrm{m}^{2}$. Ultimately, according to our new method, the Josephson penetration depth have been calculated by Eq. (1) via the "s" parameter determined in the previous section and the critical current density. The Josephson penetration depth values have been obtained to be in the order of micrometers.

\section{Conclusion}

In this chapter, the investigation of the variation of the tunneling probability in high temperature superconductors depending on the oxygen content and that of the geometry of the sample has been realized. Moreover, a new magnetic method to calculate the Josephson penetration depth reliably has been introduced. It is also shown that this work displays a correlation between SEM, XRD data and quantum chaos points of the superconducting sample establishing a bridge between the momentum and Cartesian spaces.

The determination of chaotic points has a crucial importance for technological applications of the superconductors. Hence, the prediction of the quantum chaotic points of a superconducting system enables the technologists to figure out the reliable working temperature interval for construction of superconducting devices such as bolometers, MRS and all the magnetically sensitive detectors.

Moreover, the appropriately oxygen doped and cut mercury cuprate samples have the highest Meissner transition temperature of $140 \mathrm{~K}$ ever obtained among the other superconductors prepared under the normal atmospheric pressure. Furthermore, the stability has been confirmed by SQUID measurements performed on the mercury based cuprate samples which have been kept in air for several months after being synthesized. From this point of view, the high stability and durability of the superconducting system with the highest Meissner transition temperature make the mercury cuprate family superconductors as a convenient candidate for the advanced and sensitive technological applications

\section{References}

Anderson, PW. (1958). Coherent excited states in the theory of superconductivity: Gauge invariance and the Meissner effect. Physical Review, Vol. 110, Issue. 4 827-835, ISSN: 1094-1622 (online), ISSN:1050-2947(print). 
Anderson, P.W. (1963). Plasmons, gauge invariance, and mass, Physical Reviews, Vol. 130, Issue 1 439-442, ISSN:1050-2947(print).

Aoki, R.; Murakami, H.; Kita, T.; Shirai. M.; Nishio, Y.; Svistunov, VM.; Dyachenko, A.I. \& Afanassyev, D.N. (1996). Phonon Contribution on the high $\mathrm{T}_{\mathrm{c}}$ cooper pairing investigated by tunneling spectroscopy. Physica B, Vol. 219, Issue 1-4 172-174, ISSN: 0921-4526.

Aslan, Ö. (2007). Investigation of the symmetries and the breakages in relativistic and nonrelativistic regions in high temperature superconductors. PhD Thesis Marmara University Institute of Pure and Applied Sciences.

Aslan, Ö.; Güven Özdemir, Z.; Keskin, S.S. \& Onbaşlı, Ü. (2009). The chaotic points and xrd analysis of Hg-based superconductors". Journal of Physics: Conference Series, Vol 153, Number. 1/ 012002 1-9, ISSN:1742-6596 (online), ISSN:1742-6588 (print),

Aslan Çataltepe Ö.; Güven Özdemir, Z.; Arık, M. \& Onbaşlı Ü. (2010). Strong-electroweak unification and quantum gravity achieved in chaotic regime. submitted to Chaos Solitons \& Fractals ISSN:0960-0779 (online)

Bean, C.P. (1962). Magnetization of hard superconductors. Physical Review Letters, Vol.8, Issue 6 250-253, ISSN:1079-7114 (online), ISSN:0031-9007 (print).

Bean, C.P. (1964). Magnetization of high field superconductors. Review of Modern Physics, Vol. 36 Issue 131-39, ISSN:1539-0756 (online), ISSN:0034-6861 (print).

Bogomolny, E.; Gerland, U. \& Schmit, C. (1999). Models of intermediate spectral statistics. Physical Review E, Vol. 59, Issue 2, R1315-R1318, ISSN:1550-2376 (online), ISSN:15393755 (print).

Braunish, W.; Knauf, N.; Kataev, V.; Neuhausen, S.; Grutz, A.; Kock, A.; Roden, B.; Khomskii, D. \& Wohlleben, D. (1992). Paramagnetic Meissner effect in Bi hightemperature superconductors. Physical Review Letters, Vol. 68, Issue 12 1908-1911, ISSN:1079-7114 (online), ISSN:0031-9007 (print).

Braunish, W.; Knauf, N.; Bauer, G.; Kock, A.; Becker, A.; Freitag, B.; Grutz, A.; Kataev, V.; Neuhausen, S.; Roden, B.; Khomskii, D. \& Wohlleben, D. (1993). Paramagnetic Meissner effect in high-temperature superconductors. Physical Review B, Vol. 48, Issue 6 4030-4042, ISSN:1550-235X (online), ISSN:1098-0121 (print),

Egami, T.; Chung, J.H.; McQueeney, R.J.; Yethiraj, M.; Mook, H.A.; Frost, C.; Petrov, Y.; Dogan, F.; Inamura, Y.; Arai, M.; Tajima, S. \& Endoh, Y. (2002). Electron-phonon interactions in HTSC cuprates. Physica B Vol. 316-317, 62-68, ISSN: 0921-4526.

Evangelou, S.N. \& Pichard J.L. (2000). Critical quantum chaos and the one dimensional harper model, Physical Review Letters, Vol. 84, Number 8 1643-1646, ISSN: 1079-7114 (online), ISSN: 0031-9007 (print)

Evangelou, S.N. (2001). Critical quantum chaos, Physica B, Vol. 296, 62-65, ISSN: 0921-4526.

Fossheim, K. \& Sudbo, A. (2004). Superconductivity: Physics and Applications, John Wiley \& Sons Ltd, ISBN-10: 0-470-84452-3, Great Britain.

Horovitz, B. \& Golub, V. (2002). Spontaneous magnetization and Hall effect in superconductors with broken time-reversal symmetry. Europhys Lett. Vol. 57, Number 6 892-897, ISSN:1286-4854 (online), ISSN:0295-5075 (print).

Gleick, J. (1987). Chaos: Making a New Science, Viking, ISBN: 0140092501, New York.

Gough, C.E. (1998). C-axis conductivity and the role of d-wave superconductivity and fluctuations on anisotropic high temperature superconductors. In: The Gap Symmetry and Fluctuations in High- Tc Superconductors Nato ASI Series, Series B: 
Physics vol 371, J. Bok, G. Deutscher, D. Pavuna \& S.A. Wolf (Eds.), 465-485, Plenum Press, ISBN:978-0-306-45934-4, New York.

Greiter, M. (2005). Is electromagnetic gauge invariance spontenously violated in superconductors? Annals of Physics Vol. 319, Issue 1 217-249, ISSN:0003-4916.

Güven Özdemir, Z.; Aslan, Ö. \& Onbaşl1, Ü. (2007). Calculation of microwave plasma oscillation in high temperature superconductors. In: The Seventh International Conference on Vibration Problems ICOVP 2005 Springer Proceedings in Physics, E. İnan \& E. Kırış (Eds.), 377-382, Springer, ISBN: 978-1-4020-5400-6, Dordrecht, The Netherlands.

Güven Özdemir, Z.; Aslan, Ö.\& Onbaşl1, Ü. (2009). Terahertz oscillations in mercury cuprates superconductors, Pramana-Journal of Physics, Vol. 73, No. 4 755- 763. ISSN: 03044289.

Kadowaki, K.; Kakeya, I. \& Kindo, K.(1998). Observation of the Nambu-Goldstone mode in the high-temperature superconductor $\mathrm{Bi}_{2} \mathrm{Sr}_{2} \mathrm{CaCu}_{2} \mathrm{O}_{8+d}$. Europhys Lett., Vol. 42, $203-$ 208, ISSN: 1286-4854 (online), ISSN: 0295-5075 (print).

Ketterson, J. B. \& Song S. N. (1999) Superconductivity, Cambridge University Press, ISBN:0521-56295-3 , United Kingdom.

Lawrence, W.E. \& Doniach S. (1971). Theory of Layer Structure Superconductors. In: Proceedings of the $12^{\text {th }}$ International Conference on Low Temperature Physics, E. Kanda (Ed.), 361-362, Academic Press of Japan, Kyoto.

Li, M.S. (2003). Paramagnetic Meissner effect and related dynamical phenomena. Physics Reports, Vol. 376,Iissue 3 133-223, ISSN: 0370-1573.

Nambu Y. (1960). Quasi-particles and gauge invariance in the theory of superconductivity. Phys Rev., Vol. 117 Issue 3 648-663, ISSN:1536-6065 (online).

Nambu, Y. \& Pascual, N. (1963). Self-Consistent Models Of Strong Interaction With Chiral Symmetry, Nuovo Cimento Vol. 30, 354-365.

Nielsen, A. P.; Cawthorne, A.B.; Barbara, P.; Wellstood, F.C.; Lobb, C.J.; Newrock, R.S. \& Forrester, M.G. (2000). Paramagnetic Meissner effect in multiply-connected superconductors, Physical Review B, Vol. 62, No.21 14380-14383, ISSN: 1550-235X (online).

Onbaşl1, Ü. ; Wang, Y.T.; Naziripour, A.; Tello, R.; Kiehl, W. \& Hermann, A.M. (1996). Transport properties of high Tc mercury cuprates. Physica Status Solidi B Vol. 194, 371-382, ISSN: 0370-1972.

Onbaşlı, Ü.; Öztürk, S.; \& Hasçiçek, Y. (1998). Magnetic measurement of critical currents on mercury cuprates. Applied Superconductivity Conference California, September 1998, USA.

Onbaşlı, Ü. (2000). Oxygen post-annealing at the crirtical parameters of mercury cuprates, Physica C, Vol. 332, 333-336, ISSN 0921-4534.

Onbaşlı, Ü.; Aslan, Ö. \& Güven Özdemir, Z. (2006). The super logic in the concept of probability density. In: Logic, Mathematics and Probability IV National Symposium on Probability 5-8 September 2006, Foça İzmir, 77-87, İstanbul Kültür Üniversitesi Yayınları, Yayın No: 3, ISBN: 978-975-6957-74-5, İstanbul, Turkey.

Onbaşl1 , Ü. ; Güven Özdemir, Z. \& Aslan, Ö.(2009). Symmetry breakings and topological solitons in mercury based d-wave superconductors. Chaos, Solitons \& Fractals Vol. 42, No. 4, (30 November 2009) 1980-1989, ISSN:0960-0779 (online). 
Özdemir, Z.G.; Aslan Ö. \& Onbaşlı Ü. (2006). Determination of c-axis electrodynamics parameters of mercury cuprates. Journal of Physics and Chemistry of Solids, Vol. 67 No. 1-3 (January-March 2006) 453-456 ISSN:0022-3697.

Panagopoulos, C. \& Xiang, T. (1998). Relationship between the superconducting energy gap and the critical temperature in high-Tc superconductors. Physical Review Letters, Vol. 81, 2336-2339, ISSN: 1079-7114 (online), ISSN: 0031-9007 (print).

Quigg, C. (2008). The coming revolutions in particle physics. Scientific American February 2008,46-53, ISSN:0036-8733.

Quigg, C. (2006). Particles and the standard model. In: The New Physics For The Twenty-First Century, F. Gordon (Ed.), 86-118, Cambridge University Press, ISBN-10:0-52181600-9, New York.

Rickazyen, G. (1958). Meissner effect and gauge invariance. Physical Review Vol. 111 Issue 3 817-821, ISSN: 1094-1622 (online), ISSN:1050-2947(print).

Roman, P. (2004). Why symmetry?, In: Symmetries in Science XI, B. J. Gruber, G. Marmo \& N. Yoshinaga (Eds.), 1-12, Kluwer Academic Publisher, ISBN:1-4020-2633-1 (HB), Netherlands.

Sigrist, M.; Agterberg, D.F.; Frigeri, P.A.; Hayashi, N.; Kaur, R.P.; Koga, A.; Milat, I. \& Wakabayashi, K. (2006). Unconventional superconductivity in noncentrosymmetric materials In: Effective Models for Low Dimensional Strogly Correlated Systems AIP Conf Proc Vol. 816, G.G. Batrouni \& D. Poilblanc (Eds.), 124-135, American Institute of Physics, ISBN: 0-7354-0309-0, New York.

Smolin, L. (2006). The trouble with physics, the rise of string theory, the fall of a science and what come next, Houghton Mifflin Company, ISBN-10:0-618-55105-0, Boston/New York.

Tachiki, M.; Koyama, T. \& Takahashi, S. (1996)-(a). Some exotic phenomena related to vortices in high-Tc and heavy fermion superconductors. Physica C Vol. 263, Number 1 May 1996 1-11, ISSN: 0921-4534.

Tachiki, M.; Koyama, T. \& Takahashi, S. (1996) -(b). Josephson plasma in high temperature superconductors. In: Coherence in Superconductors, G. Deutscher \& A. Revcolevschi (Eds.), 371-392, World Scientific Publishing Company Inc., ISBN-10:9810226500, Singapore.

Tinkham, M. (2004). Introduction to Superconductivity, (Second Edition) Dover Publications, ISBN-10: 0486435032 New York.

Tsudo, N. \& Shimada, D. (2003). Layer number dependence of superconducting critical temperature of cuprates: consequence of a phonon contribution to the pairing. Physica C Vol. 390, Issue 2 (15June 2003) 95-100, ISSN: 0921-4534.

Tsuei, C.C. \& Kirtley, J.R. (2000). Pairing symmetry in cuprate superconductors. Reviews of Modern Physics, Vol. 72, Issue 4 969-1016, ISSN: 1539-0756 online), ISSN: 0034 6861(print).

Ustinov, A.V. (1998). Solitons in Josephson junctions. Physica D Vol. 123, Issue 1-4 (15 November 1998) 315-329, ISSN: 0167-2789 (print).

Veltman, M.J. (1986). The Higgs bosons. Scientific American November 1986, 88-94, ISSN:0036-8733.

Waintal, X.; Weinmann, D. \& Pichard, J. L. (1999). Two interacting particles in a disordered chain II: Critical statistics and maximum mixing of the one body state. The European Physical Journal B Vol. 7 Februaury I 451-456, ISSN: 1434-6036 (online), ISSN: 14346028 (print). 
Wagner, J.L.; Hunter, B.A.; Hinks, D.G. \& Jorgensen, J.D. (1995). Structure and superconductivity of $\mathrm{HgBa} 2 \mathrm{Ca} 2 \mathrm{Cu} 3 \mathrm{O} 8+\delta$. Physical Review B Vol. 51, Issue 21 1540715414, ISSN: 1550-235X (online), ISSN: 1098-0121 (print).

Weinberg, S. (1996). The Quantum Theory of Fields Volume II, Cambridge University Press, ISBN:0-521-55002-5 (hardback), USA.

Zhang, W. (2001). Nontrivial gauge symmetry in high Tc superconductivity. Physica C, Vol. 364-365, (November 2001) 147-150, ISSN: 0921-4534. 


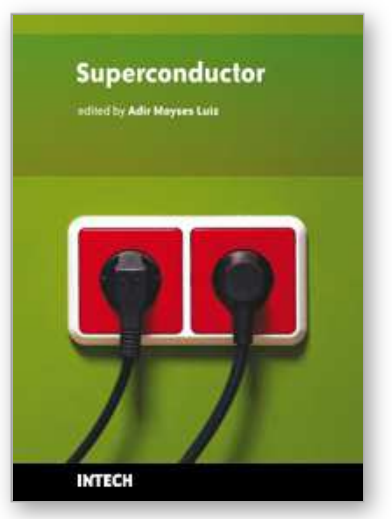

\author{
Superconductor \\ Edited by Doctor Adir Moyses Luiz
}

ISBN 978-953-307-107-7

Hard cover, 344 pages

Publisher Sciyo

Published online 18, August, 2010

Published in print edition August, 2010

This book contains a collection of works intended to study theoretical and experimental aspects of superconductivity. Here you will find interesting reports on low-Tc superconductors (materials with $\mathrm{Tc}<30 \mathrm{~K}$ ), as well as a great number of researches on high-Tc superconductors (materials with Tc> $30 \mathrm{~K}$ ). Certainly this book will be useful to encourage further experimental and theoretical researches in superconducting materials.

\title{
How to reference
}

In order to correctly reference this scholarly work, feel free to copy and paste the following:

Özden Aslan Cataltepe (2010). Some Chaotic Points in Cuprate Superconductors, Superconductor, Doctor Adir Moyses Luiz (Ed.), ISBN: 978-953-307-107-7, InTech, Available from:

http://www.intechopen.com/books/superconductor/some-chaotic-points-in-cuprate-superconductors

\section{INTECH}

open science | open minds

\section{InTech Europe}

University Campus STeP Ri

Slavka Krautzeka 83/A

51000 Rijeka, Croatia

Phone: +385 (51) 770447

Fax: +385 (51) 686166

www.intechopen.com

\section{InTech China}

Unit 405, Office Block, Hotel Equatorial Shanghai

No.65, Yan An Road (West), Shanghai, 200040, China

中国上海市延安西路65号上海国际贵都大饭店办公楼405单元

Phone: +86-21-62489820

Fax: $+86-21-62489821$ 
(C) 2010 The Author(s). Licensee IntechOpen. This chapter is distributed under the terms of the Creative Commons Attribution-NonCommercialShareAlike-3.0 License, which permits use, distribution and reproduction for non-commercial purposes, provided the original is properly cited and derivative works building on this content are distributed under the same license. 